\title{
MODELLING AND SIMULATION OF A TWO SPEED ELECTRIC VEHICLE
}

\author{
P. D. Walker ${ }^{1}$, S. Abdul Rahman ${ }^{2}$, N. Zhang ${ }^{1}$, W. Zhan ${ }^{3}$, B. Zhu ${ }^{3}$, and H. Du ${ }^{4}$ \\ ${ }^{1}$ University of Technology, Sydney, Ultimo, New South Wales, 2007, Australia \\ ${ }^{2}$ Universiti Malaysia Terengganu, Kuala Terengganu, 21030, Malaysia \\ ${ }^{3}$ Beijing Electic Vehicles, Chao Yang District, Beijing, 100021, China \\ ${ }^{4}$ Univsersity of Wollongong, Wollongong, New South Wales, 2522, Australia \\ E-mail: Paul.Walker@uts.edu.au
}

\begin{abstract}
Electric vehicles offer an alternative to hybrid and conventional vehicles through the use of electcric drive without fossil fuel consumption. This shifts green house gas production from the vehicle to power stations, but if power is supplied from renewable sources, such as wind power, zero emissions are generated. Practical vehicle design requires the consieration of competing demands of vehicle acceleration and performance against range and vehicle efficiency. Thus, considering requirements such as grade climbing and acceleration against vehicle range and power consumption, a two speed transmission is suggested for this vehicle study to increase motor operation at high torque and efficiency regions. To evaluate the application of such a transmission, a two speed electric vehicle powertrain is developed in Simulink®. Simulations are conducted to demonstrate the performance of the two speed electric vehicle. Results reveal the capability of the two speed vehicle to meet various performance criteria and provide an indication of effective range under different drive cycles.
\end{abstract}

\section{Introduction}

Pure electric vehicles (EV) have become an alternative to hybrid electric vehicles and conventional passenger vehicles as they produce zero emissions on the road. Such vehicles rely on significantly higher energy in battery cells compared to the hybrid equivalent to produce reasonable vehicle range. For example the $1100 \mathrm{~kg}$ iMIEV compact EV has arrange of $130 \mathrm{~km}$ on $16 \mathrm{kWh}$ (Kamachi, Miyamoto, \& Sano 2010), whereas the all electric range of the Chervrolet Volt, weighing in at $1715 \mathrm{~kg}$ is less than half that at $60 \mathrm{~km}$ (Brooke 2011), though the overall range is closer to $500 \mathrm{~km}$. To therefore provide a reasonable range capability for a full sized EV sedan, evaluation of performance capabilities against design range is required to ensure correct size of batteries, motor and reduction ratio in the vehicle. 
Vehicle drive cycles are frequently used to evaluate vehicle range under "normal" driving conditions, generally defined as either highway or urban drive cycles. Examples include Abdul Rahman, Zhang, \& Zhu (2008), studying energy management for series-parallel HEVs with a planetary transmission. Driving simulations are then used to evaluate energy management, storage, and fuel consumption. For EVs energy management is reduced to acceleration and braking requirements, reducing complexity, however the energy storage system becomes critical to the vehicle. While there is a push to super-capacitors, electric vehicles rely on large battery modules to achieve range (Jinnui, Zhifu, \& Qinglian 2006).

The application of multispeed transmissions for EVs has the potential to improve average motor efficiency and hence range, or even reduce the required motor size. There is a range of transmissions available for application to EVs for multispeed drives, in Rudolph, et al, (2007) it is suggested that DCTs have higher efficiency than other automatic drives, making them particularly suitable.

This paper presents a systematic model of a two speed EV for evaluation of vehicle range and performance with an integrated two speed transmission. In the following sections are divided into EV powertrain model development in Section 2 , including electric machine, battery, transmission, vehicle, and driver models. This is followed by vehicle performance evaluation and range simulations using two drive cycles. Finally, conclusions are drawn and project direction identified.

\section{EV powertrain modelling}

The main components of the electric vehicle power train that are considered for modeling are as follows:

- electric machine

- battery pack

- transmission and vehicle

- Driver model.

\subsection{Electric Machine Model}

Electric machines (EM) in electric vehicles have a dual role, providing driving torque to the vehicle and recovering energy during braking events. However, EM are frequently designed to provide higher efficiency as either a motor or generator. This leads to limitations in implementing the EM as a generator. It is modelled using look up tables to define motor efficiency as a function of speed and input power, while the actual output torque is defined from the input power and motor speed, limited by the rated motor torque. When acting as a generator it is assumed 
that only $30 \%$ of the generated power can be used to charge the battery, and for a vehicle speed under $15 \mathrm{kph}$ the generator is ineffective and dissabled, friction brakes are then only used for braking. From energy conservation, electric machine torque is:

$$
T_{E M}=\eta_{E M} \eta_{P C} \frac{P_{B}}{\omega_{E M}}
$$

\subsection{Battery model}

The battery model is based on lithium-ion batteries using interpolation to evaluate battery characteristics, similar to the model described in Abdul Rahman, Zhang, \& Zhu (2008). Requirements are to determine cell characteristics $\left(\mathrm{R}_{\mathrm{INT}}\right.$ and $\left.\mathrm{V}_{\mathrm{OC}}\right)$ as a function of temperature and SOC, and calculate the output voltage, battery temperature and change of SOC, all based on the input current to the battery. The output voltage of the battery during charge or discharge is:

$$
V_{O U T}=V_{O C}-R_{I N T} \times I
$$

Maximum capacity is determined from the battery temperature $\left(\mathrm{CAP}_{\mathrm{MAX}}\right)$, and used capacity $\left(\mathrm{CAP}_{\mathrm{USED}}\right)$ from the initial conditions and supply/demand from the motor/generator. The absolute state of charge $\left(\mathrm{SOC}_{\mathrm{ABS}}\right)$ is defined as:

$$
S O C_{A B S}=\frac{C A P_{M A X}-C A P_{U S E D}}{C A P_{M A X}}
$$

\subsection{Transmission and vehicle models}

The purpose of vehicle transmissions is to provide maximum vehicle performance and efficiency using many ratios. For an EV the transmission is based on a DCT with only two gears, with shifting controlled using a shift map designed to promote the operation of the EM in the higher efficiency region. Detailed ratio design is available in Lechner \& Naunheimer (1999) for grade and speed requirements. Here maximum grade climbing is used to evaluate lowest possible ratio, while top speed and driving torque are used to limit the top gear ratio.

The vehicle model takes all the input torques, calculates vehicle acceleration and performs numerical integration to determine vehicle speed. Inputs are 
supplied motor torque, $\mathrm{T}_{\mathrm{EM}}$, brake torque, $\mathrm{T}_{\mathrm{B}}$, and vehicle resistance torque, $\mathrm{T}_{\mathrm{V}}$, and the output is vehicle acceleration. Equation of motion for the vehicle is:

$$
m_{V} r_{t}^{2} \alpha=\eta_{P T} \gamma T_{E M}-T_{V}-T_{B}
$$

The vehicle resistance torque is the combination of rolling resistance force, incline load and air drag. It is defined as:

$$
T_{v}=\left(C_{R} m_{V} g \cos \phi+m_{V} g \sin \phi+0.5 C_{D} \rho A_{V} \omega_{V}^{2} r_{r}^{2}\right)
$$

\subsection{Driver model}

The vehicle driver is modeled using a PID controller, where the difference between demanded drive cycle speed and actual vehicle speed is the input error. The PID output is demand power to drive the vehicle, where a positive signal is considered the power demand required for vehicle acceleration, and a negative demand is the power required for braking.

\section{Simulations and analysis}

The purpose of performing simulations is to provide a demonstration of the performance capabilities of the proposed vehicle. Simulations are conducted to evaluate the driving criteria of range under one highway and one urban style drive cycle, acceleration, overtaking acceleration, and grade climbing. The vehicle specifications are summarized in Table 1.

Table 1 Vehicle parameters

\begin{tabular}{|lr|lr|}
\hline \multicolumn{2}{|c|}{ Parameter } & \multicolumn{2}{c|}{ Parameter } \\
\hline Mass & $1760 \mathrm{~kg}$ & Battery Layout & $1 \mathrm{P} 120 \mathrm{~S}$ \\
\hline Wheel radius & $0.266 \mathrm{~m}$ & Battery Voltage & $384 \mathrm{~V}$ \\
\hline Frontal area & $2.2 \mathrm{~m}^{2}$ & Battery Capacity & $66 \mathrm{Ah}$ \\
\hline Rolling resistance & 0.016 & Gear ratios & $8.68,5.7$ \\
\hline Motor torque & $255 \mathrm{Nm} @ 3000$ RPM (peak) 127 Nm @ 9000 RPM \\
\hline
\end{tabular}

The first cycle is a highway style drive, known as Highway Fuel Economy Test (HWFET), with repeated long high speed cycles to simulate highway driving. The second cycle is Urban Dynamometer Driving Schedule (UDDS), designed to simulate city driving with repeated stop starts. 
The modeled vehicle is capable of $0-100 \mathrm{kph}$ in $13.7 \mathrm{~s}$, overtaking acceleration of $50-80 \mathrm{kph}$ in $4.7 \mathrm{~s}$, and grade climbing of over $30 \%$. Figure 1 below shows vehicle response to HWFET drive cycle, while Figure 2 shows the vehicle response to a UDDS cycle. A single cycle vehicle speed is shown in (a), while (b) shows the gear ratio changes for both figures. The vehicle range with the HWFET drive cycle is $141.2 \mathrm{~km}$, equivalent to approximately 1.8 hours running time, a short but reasonable distance for an EV. By comparison the vehicle range under the UDDS cycle, Figure 2, is $128.2 \mathrm{~km}$, a total drive time of about 4 hours. The longer range using HWFET is realized by the vehicle running continuously at a relatively stable speed with low torque demand. The UDDS cycle range is extended by frequent application of regenerative braking.

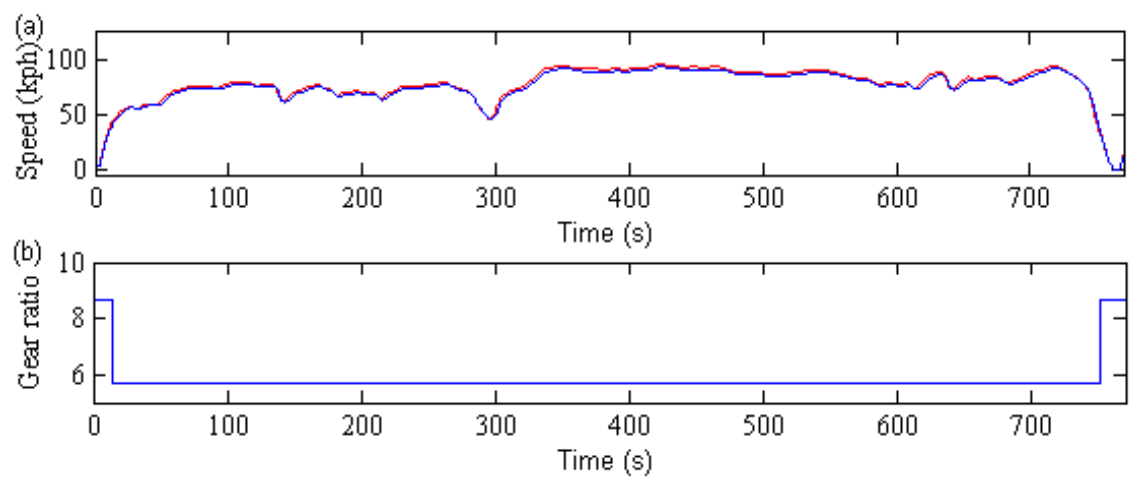

Fig. 1 Simulation results for HWFET cycle (a) drive cycle and (b) gear ratio.

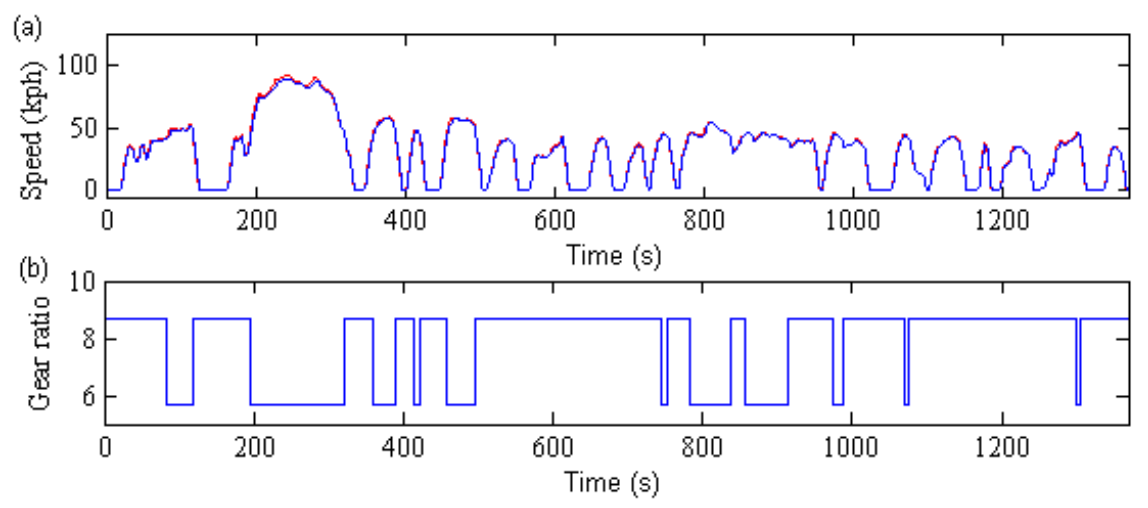

Fig. 2 Simulation results for UDDS cycle (a) drive cycle and (b) gear ratio.

Results in Figure 3 demonstrate the operating locus of the electric machine for both drive cycles. The results using only first gear demonstrate operation at higher speeds, with low efficiency. However, the employment of a two speed transmission shows improved efficiency by reducing operation in low efficiency zones. 

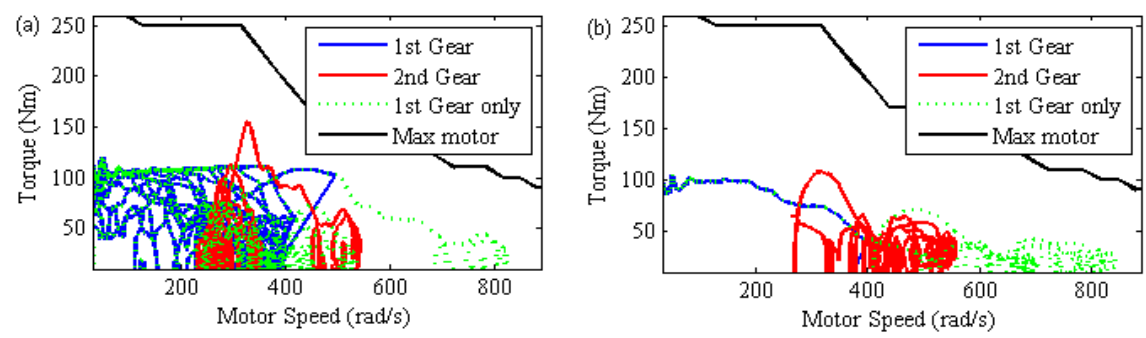

Fig. 3 Motor torque-speed traces for (a) UDDS cycle and (b) HWFET cycle.

\section{Conclusion}

This paper presented a compact model of a two speed electric vehicle to be used for performance evaluation in the Matlab/Simulink® environment. Simulations were conducted to evaluate performance specifications and range simulations. The results demonstrate that the larger vehicle is capable of meeting several performance criteria for acceleration and grade climbing, and provides a reasonable range for a large EV. Continued research for this project will focus on component optimization, shift dynamics, and significant experimental validation.

\section{Acknowledgements}

This project is supported by the Beijing Electric Vehicle Company and Ministry of Science and Technology, China.

\section{References}

Kamachi M. Miyomoto H. and Sano Y. (2010). Development of Power Management System for Electric Vehicle 'iMIEV' 2010 International Power Electronics Conference, pp. 2949 - 2955 Brooke L. (2011) Chevrolet Volt: AEI Best Engineered Vehicle AEI-Online.org, pp. 22 - 25.

Abdul Rahman S. Zhang N. \& Zhu J. (2008). Optimal Energy Management for Plug-in Hybrid Electric Vehicles, $3^{\text {rd }}$ International Conference on Mechatronics pp. 1-6.

Jinrui N. Zhifu W. Qinglian R. (2006). Simulation and Analysis o Perofrmance of a Pure Electric Vehicle with a Super-capacitor Vehicle Power \& Propulsion Conference. pp 1-6.

Rudolph F. Schafer M. Damm A. Metzner F T. and Steinberg I. (2007) The Innovative Seven Speed Dual Clutch Gearbox for Volkswagen's Compact Cars $28^{\text {th }}$ Internationales Wiener Motorensymposium

Lechner G. and Haunheimer H. (1999). Automotive Transmissions: Fundamentals, Selection, Design, and Application, Springer, Germany. 